\title{
Birth of a healthy infant after bone marrow-derived cell therapy
}

\author{
Nayana H Patel, Yuvraj D Jadeja, Niket H Patel, Molina N Patel, Harsha K Bhadarka, Piyush N Chudasama, Harmi R Thakkar \\ Department of Reproductive Medicine, Akanksha Hospital and Research Institute, Anand, India
}

Bone marrow-derived cell (BMDC) therapy has numerous applications as potential biological cells for use in regenerative medicine. Here, we present an original case of endometrial atrophy associated with genital tuberculosis in a woman who achieved a live birth with BMDC. This 27-year-old woman came to our center with endometrial atrophy and primary infertility. She had a past history of genital tuberculosis and amenorrhea. Her husband's semen quality was normal. The patient was counseled for hysteroscopy due to thin endometrium and advised in vitro fertilization (IVF) with donor eggs in lieu of poor ovarian reserve. Several attempts of IVF with hormone replacement therapy (HRT) were made, but the desired thickness of the endometrium was not achieved. Uterine artery injection of BMDC through interventional radiology was given, followed by HRT for three months, which resulted in improved endometrium. This was subsequently followed by IVF with donor egg. The treatment resulted in the conception and delivery of a 3.1-kg baby boy through lower segment caesarean section with no antenatal, intranatal or postnatal complications. Recently, there has been massive interest in stem cells as a novel treatment method for regenerative medicine, and more specifically for the regeneration of human endometrium disorders like Asherman syndrome and thin endometrium, which was the reason behind using this strategy for treatment.

Keywords: Asherman's syndrome; Bone marrow-derived cell therapy; Endometrial atrophy; Regenerative medicine; Stem cell therapy

\section{Introduction}

The human endometrium is a dynamic remodeling tissue that undergoes more than 400 cycles of growth, differentiation, and shedding of endometrial cells during the reproductive period [1]. Asherman syndrome (AS) is an uncommon gynecological disorder caused by the destruction of the endometrium due to repeated or aggressive curettages and/or endometritis. As a result, there is a loss of functional endometrium in many areas, and the uterine cavity is obliterated by intrauterine adhesions, leading to amenorrhea, hypomenorrhea, infertility, recurrent pregnancy loss, and/or abnormal placentation, including placenta previa and accrete [2,3].

Endometrial atrophy $(\mathrm{EA})$ is another rare condition in which the

Received: November 24, 2020 • Revised: May 13, 2021· Accepted: May 17, 2021 Corresponding author: Nayana H Patel

Department of Reproductive Medicine, Akanksha Hospital and Research Institute, Near Srishti English Medium School, Lambhvel, Anand, Gujarat 387310, India Tel: +91-98-2515-8227 E-mail: nayana@ivf-surrogate.com

This is an Open Access article distributed under the terms of the Creative Commons Attribution Non-Commercial License (http://creativecommons.org/licenses/by-nc/4.0/) which permits unrestricted non-commercial use, distribution, and reproduction in any medium, provided the original work is properly cited. endometrium is too thin and never grows more than $5 \mathrm{~mm}$ thick. Factors that can cause EA include prolonged use of oral contraceptives and Tamoxifen. Genital tuberculosis (TB) affects endometrial receptivity leading to defective endometrial markers and EA. The prevalence of this pathology is $0.5 \%$ in infertile women undergoing assisted reproductive treatments (ARTs) [2].

Successful implantation in ART requires a high-quality embryo, receptive endometrium, and perfect embryo transfer technique. Current treatment strategies for AS aim to break up the synechiae and induce endometrial proliferation in order to restore functionality to the uterine cavity. Unfortunately, the risk of treatment failure is high. These fibrotic synechiae often lack endometrial lining entirely, or, if present, the lining is thin and largely nonfunctional [4].

Evidence suggests that adult stem cell populations exist in the human endometrium. Hence it might be possible to activate endogenous endometrial stem/progenitor cells in cases of atrophic or thin endometrium or to transplant bone marrow-derived cells (BMDCs) in the uterine lining for endometrial regeneration in $\mathrm{AS}$ or severe cases of intrauterine adhesions. Low levels of circulating BM-derived hemopoietic stem cells, mesenchymal stem cells, and endothelial progeni- 
tor cells integrate into damaged tissues and trans-differentiate into host tissues, including the endometrium. Evidence is available where $B M D C$ has been used to treat AS and EA in an attempt to achieve normal reproductive functioning of the endometrium $[1,5,6]$.

\section{Case report}

A 27-year-old female presented to our center on May 15, 2017. Her husband's age was 29 years, and they had been married for the last 4 years. The patient gave a history of secondary amenorrhea for 2 years and she informed that her menstrual cycle was very irregular (just drops during her periods) but denied any abdominal pain or cramps during this time. Her last menstrual period (LMP) was April 8, 2017.

Previous medical history revealed that she had genital TB 2 years before presentation to our clinic. TB was diagnosed by laparohysteroscopy done at another center, which revealed tubercles in the whole abdomen. Her husband's semen analysis was found to be normal at 46 million counts, and motility was 54\%. Transvaginal sonography was performed, which revealed that the endometrial lining was very thin with fluid ( $<5 \mathrm{~mm}$ ) (Figure 1$)$. She was given estradiol 2-mg tablet for 10 days but there was no improvement in the lining of the endometrium (Figure 2). On May 29, 2017, hysteroscopy was performed on the 14th day of the cycle, which showed that the cervix was stenosed and the uterus was acutely anteverted. Bands of adhesions were seen on the fundus and lateral walls (Figure 3). The fibrotic bands of adhesions were cut, and lateral metroplasty was done to enlarge the cavity. Hormone replacement therapy (HRT) was continued, and progesterone (10 mg/day) was added on June 18 for 5 days. The patient got withdrawal bleeding (very scanty on July 8).

Second attempt to prepare the endometrium was made in July with HRT, which comprised of Estradiol $2 \mathrm{mg}$, Norethisterone along with the addition of Estrogel three times a day (subcutaneous estro-

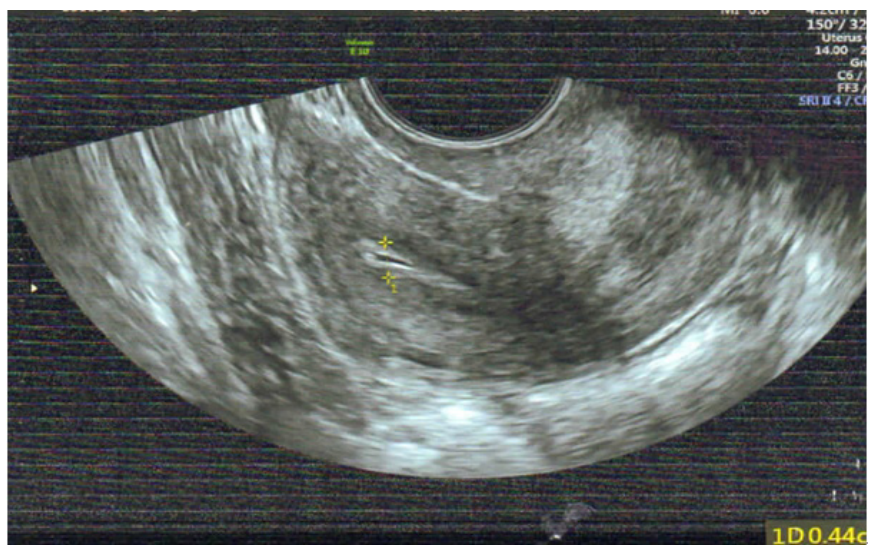

Figure 1. Transvaginal sonography showing endometrial lining was very thin with fluid $(<5 \mathrm{~mm})$. gen supplementation) and Aspirin $75 \mathrm{mg}$ three times a day. Endometrial thickness was measured on the 8th day, which was $4.7 \mathrm{~mm}$. On the 15th day, it was observed that the endometrium was patchy, and endometrial thickness was $5.5 \mathrm{~mm}$. Progesterone was given to induce bleeding. She had scanty bleeding on the 14th of August.

Endometrial preparation was again tried with HRT plus adjuvants such as Arginitric sachet and Sildenafil. On August 30, 2017, the ultrasound showed an endometrial thickness of $4.3 \mathrm{~mm}$ with endometrial fluid. So, on September 5, 2017, a relook hysteroscopy was done, which revealed no adhesion in the cavity, and the endometrium was

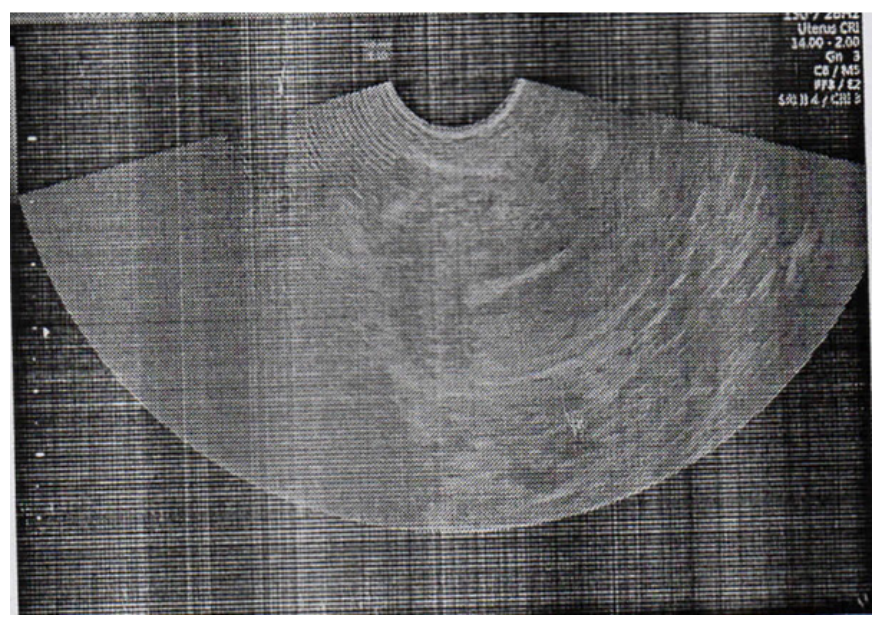

Figure 2. Transvaginal sonography showing thin echogenic line and no improvement in lining after hormone replacement therapy.

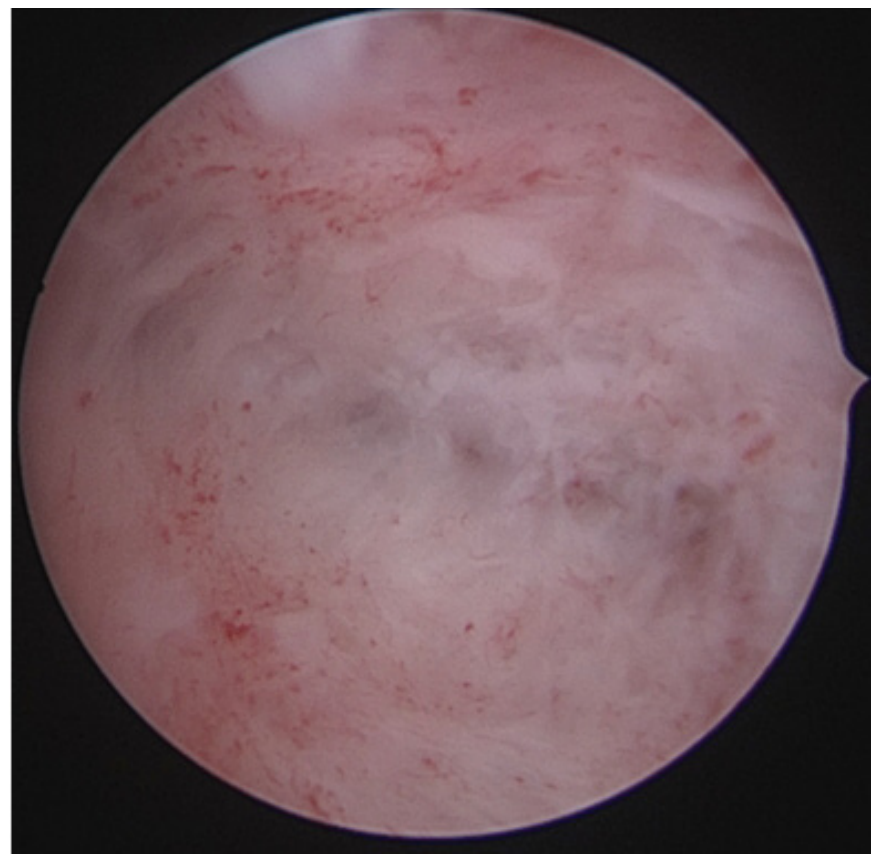

Figure 3. Hysteroscopy showing fibrotic bands in uterine cavity. 
found to be pink (Figure 4). The patient did not come to the clinic for 3 months after this visit.

The patient reported to the center again on December 25, 2017, with LMP on December 20, 2017 (scanty bleeding). Endometrial preparation was tried with gonadotropin stimulation (injection HMG $75 \mathrm{mg}$ ) plus adjuvants such as Arginitric sachet and Sildenafil. On the 13th day, the endometrial thickness was $5.5 \mathrm{~mm}$, and one follicle was seen on the left side of size $20 \mathrm{~mm}$. Human chorionic gonadotropin (HCG) trigger was given on the 15th day. On the day of embryo transfer, endometrial thickness was $5.8 \mathrm{~mm}$ with a patchy endometrium. Embryo transfer was done, but with negative results.

The patient stopped all treatment after that visit and came back after 1 year. It was observed on studying the records that despite giving HRT, gonadotropin and adjuvants) multiple times, the endometrial thickness was not achieved. So, the patient was counseled about the BMDC therapy for which she gave her consent.

\section{Pre-procedure workup for BMDC}

The patient was admitted on January 16, 2019, early morning and pre-procedure workup was commenced with injection Ceftriaxone 1g IV statim, $\mathrm{O}_{2} @ 2-4 \mathrm{~L} / \mathrm{min}$ for 30 minutes, injection vitamin C150 $\mathrm{mg}$ (diluted in $100 \mathrm{~mL}$ normal saline [NS]), and injection glutathione $600 \mathrm{mg}$ (diluted in $100 \mathrm{~mL} \mathrm{NS}$ ).

\section{Collection of bone marrow aspirate}

The patient was made to lie down in the horizontal position. The

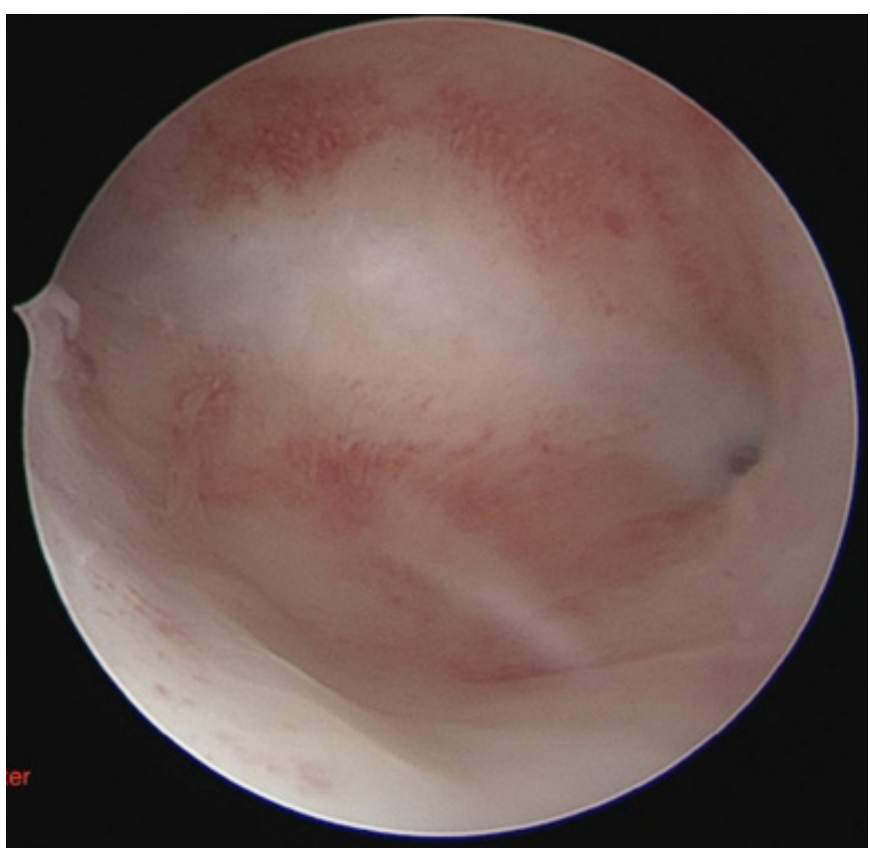

Figure 4. Relook hysteroscopy showing endometrium without adhesion. procedure began 1 hour later on the same day with sedation of the patient with intravenous Midazolam and Fentanyl. Approximately, $60 \mathrm{ml}$ of bone marrow aspirate (BMA) was collected over acid citrate dextrose-anticoagulant $(5 \mathrm{~mL})$ from the patient's anterior superior spine of the iliac crest. The position of the Jamshidi needle was confirmed by loss of resistance after piercing the compact bone. BMA was collected in a $60 \mathrm{~mL}$ syringe in a series of discrete pulls on the plunger (targeting a collection of 5-10 mL per pull) with repositioning of the needle tip between pulls based on the reported enrichment of progenitor cells.

\section{Preparation of BMDC}

About $10 \mathrm{~mL}$ of diluted BMA (1:2 with sterile phosphate buffer saline [PBS]) was aseptically transferred to a $15.0 \mathrm{~mL}$ sterile centrifuge on top of a $3.0 \mathrm{~mL}$ density gradient solution. The tube was then centrifuged at $2000 \mathrm{RPM}$ for 30 minutes at room temperature $\left(15^{\circ} \mathrm{C}-25^{\circ} \mathrm{C}\right)$. This centrifugation step eliminated erythrocytes and poly leukocytes from the mixture of cells and a sharp band appeared containing mononuclear cells (BMDC). BMDC was then collected in an aseptic tube, washed with PBS twice, and counted using a hemocytometer. Cell viability was analyzed using trypan blue assay, and then cells (15 million cells $/ \mathrm{mL}$ ) were prepared for administration to the patient without any further manipulations.

\section{Intra-arterial injection}

Two hours after BMA and proper consent, the patient was taken for intra-arterial injection into the catheterization laboratory. Under aseptic precautions, bilateral femoral artery access was taken with 6-Fr sheath via the Seldinger technique under local anesthesia. Six-Fr diagnostic JR catheter was used to reach the contralateral internal iliac artery over a 0.035 -inch Terumo guide wire. It was then exchanged for 6-Fr JR 3.5 guiding catheter over 0.035 -inch stiff wire. The uterine artery was identified by contrast dye injection in the internal iliac artery. Super selective cannulation of the uterine artery and subsequently spiral arterioles was done with a microcatheter over 0.014-inch PTCA workhorse wire. On January 16, 2019, uterine artery spiral arterioles BMDC treatment was performed. Patency of artery post-injection was confirmed by contrast dye injection from guide catheter through the internal iliac artery. Post-procedure femoral sheaths were removed in the catheterization laboratory with manual compression, and lower limb immobilization was done in the supine position for 6 hours in the recovery room. The patient was slowly mobilized under observation and discharged the next day.

\section{Post-procedure follow-up}

The patient was put on HRT for 3 months consisting of sequential estrogen and progesterone therapy. In April, we checked her uterine 
lining with HRT and the serial scan showed good triple line and improved thickness (Figure 5). The endometrium was monitored every month until the month of frozen embryo transfer (FET). The patient showed an increase in the volume of menstrual blood flow for three cycles. Description of menstrual flow after the therapy is described in Table 1.

\section{Frozen embryo transfer}

Formation of embryos with donor eggs was performed. A total of 10 eggs were retrieved from a healthy donor of 31 years of age, fertilized with the patient's sperm sample. Four blastocysts were formed for prospective embryo transfer. FET was performed in May, endometrial preparation was done by HRT comprising of tablet Estradiol valerate $2 \mathrm{mg}$, Estrogel thrice a day, Arginitric sachet thrice a day and Aspirin $75 \mathrm{mg}$. On the 8th day of HRT, endometrial thickness was 5.4 $\mathrm{mm}$, and on day 11, it was found to be $7.1 \mathrm{~mm}$. Post-bone marrow-derived stem cells (BMDSC) in the endometrium showed improved response to HRT in terms of the thickness and echo texture both (Figure 6). Color doppler was favorable. Injection progesterone for 5 days was added for blastocyst transfer. The blastocyst was frozen under Kitazato vitrification and warming protocol before transfer.

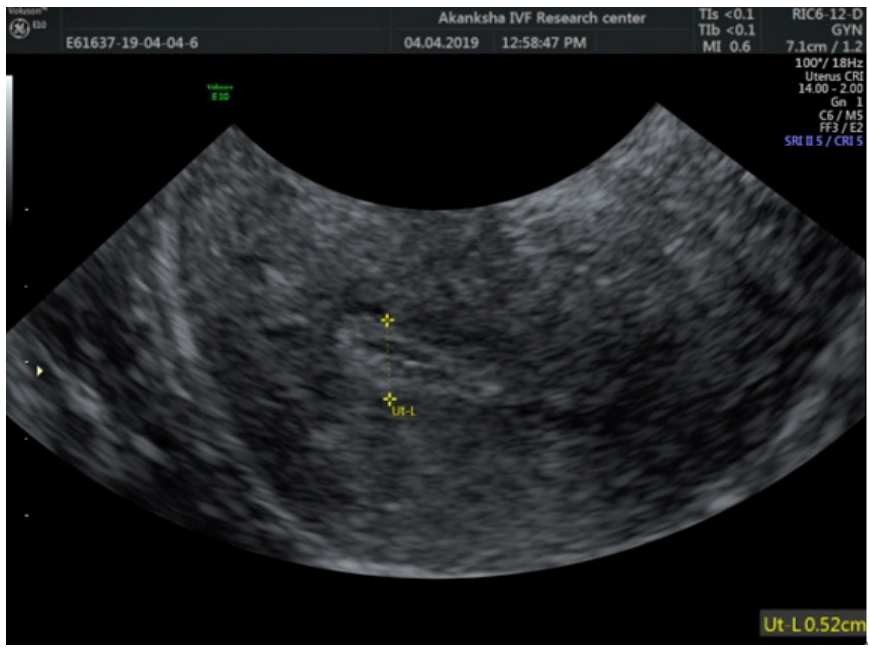

Figure 5. Post-bone marrow-derived stem cells: the response to hormone replacement therapy showed drastic improvement in lining, thickness as well as echo texture in the April 2019.

Table 1. Description of menstrual flow after the therapy

\begin{tabular}{lc}
\hline Date & No. of pads used \\
\hline January 28, 2019 & $2-3$ \\
February 26, 2019 & $3-4$ \\
March 27, 2019 & 4 \\
April 24, 2019 & 4 \\
May 13, 2019 & $3-4$ \\
\hline
\end{tabular}

On May 30, 2019, the embryo transfer was performed smoothly without any difficulty. Only one blastocyst of grade 3AA was transferred. Luteal phase support was given, and beta HCG was tested on June 14,2019 , which turned out to be $1,212 \mathrm{mg} / \mathrm{dL}$. Doubling titer repeated on July 16,2019 , was $3,286.6$, confirming a positive result. The first scan was performed at around 7 weeks that showed single live intrauterine pregnancy (Figure 7). The antenatal period was uneventful, except for an episode of preterm pain at around 24 weeks. Planned lower segment caesarean section (LSCS) was performed after 38 weeks of gestation. A 3.1-kg baby boy was delivered, and LSCS was uneventful.

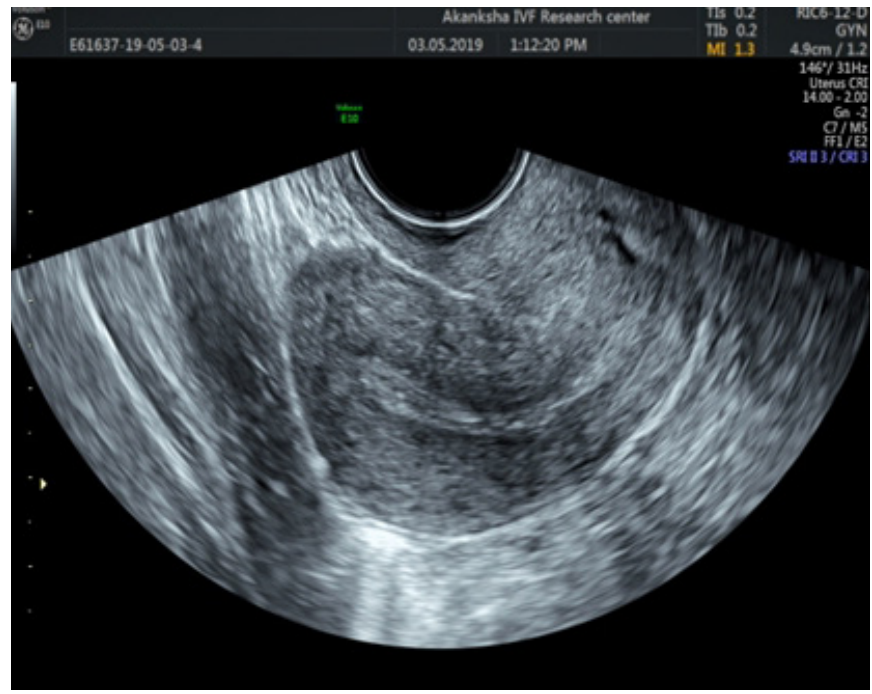

Figure 6. Post-bone marrow-derived stem cells: the response to hormone replacement therapy showed drastic improvement in lining, thickness as well as echo texture in May 2019 when frozen embryo transfer was done.

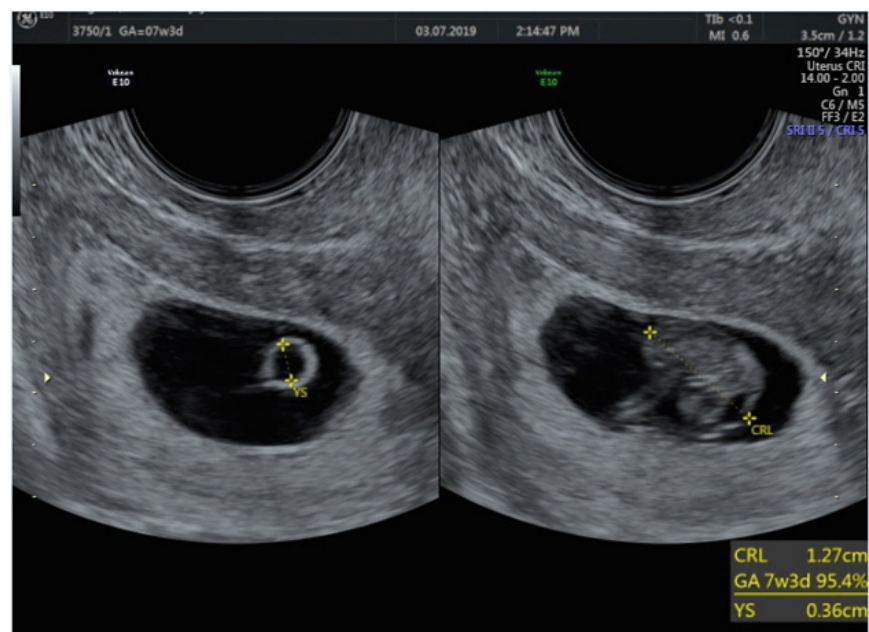

Figure 7. Scan showing single live intrauterine pregnancy. 


\section{Discussion}

Adult bone marrow is a reservoir of stem and progenitor cells. BMDCs can trans differentiate into multiple non-hematopoietic cell lineages, and play a role in the reconstitution of the human endometrium. Clinical studies suggest that ischemic/reperfusion injury provides a strong stimulus for homing and engraftment of BMDCs into the uterus and it has been suggested that one of the mechanisms by which uterine injury may improve endometrial receptivity is via increasing recruitment of BMDCs to the endometrium. Systemic administration of BMDCs can improve uterine scar healing and fertility in AS [7]. Santamaria et al. [2] conducted a clinical study to analyze the effect of BMDSC in patients with refractory AS and/or EA. BMDSC mobilization was performed by granulocyte colony-stimulating factor injection in a total of 16 patients. Endometrial thickness increased from an average of $4.3 \mathrm{~mm}$ to $6.7 \mathrm{~mm}$. Similarly, four of the five EA patients experienced an improved endometrial cavity, and endometrial thickness increased from $4.2 \mathrm{~mm}$ to $5.7 \mathrm{~mm}$ [2].

Another study was conducted by Cervello et al. [8] using the engraftment of BMDSC predominantly around the endometrial blood vessels of traumatized endometrium. Results revealed that BMDSCs induces proliferation of the neighboring endometrial cells in the damaged endometrium, mainly at the epithelial component [8]. Azizi et al. [9] investigated whether BMDC treatment could improve the restoration of the endometrium and increase the endometrial receptivity. A thin endometrium model was designed, and BMDC was transplanted by tail vein injection. Results revealed that BMDC has a valuable effect on the thin endometrium, and may play a role through migration and immunomodulatory effect of BMDC [9].

To the best of our knowledge, this was one of the first successful cases of treating endometrial atrophy with BMDC leading to a living birth. This rare and novel form of treatment paves a way for future research in the treatment of endometrial factors of infertility by regenerative medicine.

\section{Conflict of interest}

No potential conflict of interest relevant to this article was reported.

\section{Acknowledgments}

Editorial support was provided by Dr. Manish Varma and Dr. Ravneet Gulshan from Spirant Communication Private Limited.

\section{ORCID}

Nayana H Patel

https://orcid.org/0000-0003-2650-2458

\section{Author contributions}

Conceptualization: all authors. Data curation: YDJ, MNP, HKB, HRT. Formal analysis: NHP, YDJ, NHP, MNP, HKB. Methodology: NHP, YDJ, HKB, PNC. Project administration: all authors. Visualization: NHP, YDJ, HKB, PNC. Writing-original draft: NHP, YDJ, MHP, HRT. Writing-review and editing: all authors.

\section{References}

1. Lee YJ, Yi KW. Bone marrow-derived stem cells contribute to regeneration of the endometrium. Clin Exp Reprod Med 2018;45: 149-53.

2. Santamaria X, Cabanillas S, Cervello I, Arbona C, Raga F, Ferro J, et al. Autologous cell therapy with CD133+ bone marrow-derived stem cells for refractory Asherman's syndrome and endometrial atrophy: a pilot cohort study. Hum Reprod 2016;31:1087-96.

3. Dreisler E, Kjer JJ. Asherman's syndrome: current perspectives on diagnosis and management. Int J Womens Health 2019;11:191-8.

4. Gargett CE, Healy DL. Generating receptive endometrium in Asherman's syndrome. J Hum Reprod Sci 2011;4:49-52.

5. Singh N, Mohanty S, Seth T, Shankar M, Bhaskaran S, Dharmendra S. Autologous stem cell transplantation in refractory Asherman's syndrome: a novel cell based therapy. J Hum Reprod Sci 2014;7: 93-8.

6. Sakr S, Naqvi H, Komm B, Taylor HS. Endometriosis impairs bone marrow-derived stem cell recruitment to the uterus whereas bazedoxifene treatment leads to endometriosis regression and improved uterine stem cell engraftment. Endocrinology 2014;155: 1489-97.

7. Liu Y, Tal R, Pluchino N, Mamillapalli R, Taylor HS. Systemic administration of bone marrow-derived cells leads to better uterine engraftment than use of uterine-derived cells or local injection. J Cell Mol Med 2018;22:67-76.

8. Cervello I, Gil-Sanchis C, Santamaria X, Cabanillas S, Diaz A, Faus A, et al. Human CD133(+) bone marrow-derived stem cells promote endometrial proliferation in a murine model of Asherman syndrome. Fertil Steril 2015;104:1552-60.e1-3.

9. Azizi R, Aghebati-Maleki L, Nouri M, Marofi F, Negargar S, Yousefi M. Stem cell therapy in Asherman syndrome and thin endometrium: stem cell- based therapy. Biomed Pharmacother 2018;102:33343. 